\title{
Numerical analysis of gradient index lens-based optical coherence tomography imaging probes
}

\author{
Woonggyu Jung \\ University of Illinois at Urbana-Champaign \\ Beckman Institute for Advanced Science and Technology \\ Urbana, Illinois 61801
}

\section{Wladimir Benalcazar \\ Adeel Ahmad \\ University of Illinois at Urbana-Champaign \\ Beckman Institute for Advanced Science and Technology \\ Department of Electrical and Computer Engineering \\ Urbana, Illinois 61801}

\author{
Utkarsh Sharma \\ Haohua Tu \\ University of Illinois at Urbana-Champaign \\ Beckman Institute for Advanced Science and Technology \\ Urbana, Illinois 61801
}

\section{Stephen A. Boppart}

University of Illinois at Urbana-Champaign

Beckman Institute for Advanced Science and Technology

Department of Electrical and Computer Engineering

and

Department of Bioengineering

Urbana, Illinois 61801

\begin{abstract}
We report the numerical analysis of gradient index (GRIN) lens-based optical coherence tomography imaging probes to derive optimal design parameters. Long and short working distance probes with a small focal spot are considered. In each model, the working distance and beam waist are characterized and compared for different values of length and refractive index of the probe components. We also explore the influence of the outer tubing and refractive index of the sample media. Numerical results show that the adjustment of the maximum beam diameter and focusing angle at the end of the GRIN lens surface is very important for determining the optical performance parameters of the probe. @ 2010 The International Society for Optical Engineering. [DOI: 10.1117/1.3523374]

Keywords: optical coherence tomography; endoscopic imaging probe; gradient index lens.

Paper 10231R received May 1, 2010; revised manuscript received Oct. 12, 2010; accepted for publication Oct. 18, 2010; published online Dec. 30, 2010.
\end{abstract}

\section{Introduction}

Optical coherence tomography (OCT) is a cross-sectional imaging modality that provides structural information of biological tissue in real time with high resolution and in a noninvasive manner. ${ }^{1}$ Over the last decade, OCT techniques have improved image acquisition speeds, and sensitivity to provide high-resolution in vivo cross-sectional or volumetric imaging of living tissue, thereby enabling applications in real-time detection of early diseases and surgical guidance. ${ }^{2}$ The miniaturization of optical imaging probes has played an important role in the enhancement of OCT for in vivo animal and human studies. ${ }^{3-6}$ Miniaturized probes can counter the limited penetration depth of OCT imaging to some extent by making it feasible to access deep tissues or organs. Endoscopic and intracoronary imaging may enable significant clinical breakthroughs in OCT imaging after the initial success of OCT in ophthalmic imaging.

Numerous types of miniaturized optical probes have been developed. These are usually designed for a specific target application with specific requirements. ${ }^{6}$ The most common miniaturized OCT probes are composed of a single-mode optical fiber (SMF), a gradient-index (GRIN) lens, and a small prism to deflect the light into the tissue of interest. The GRIN lens is a key component that determines performance of the probe, and these

Address all corresponding to: Stephen A. Boppart and Woonggyu Jung, University of Illinois Urbana-Champaign, Beckman Institute, 405 North Mathews Avenue, Urbana, Illinois 61801. E-mail: boppart@illinois.edu and wgjung@illinois.edu. have been used as an alternative to conventional bulky lenses with several advantages. The light in the GRIN lens follows a continuous curved trajectory due to the radial refractive index profile while maintaining the essential lens function in limited cylindrical spaces. Thus, the first merit of GRIN lenses is their compact form. Second, unlike conventional lenses, GRIN lenses have flat surfaces at both ends, which facilitate high quality joints between the lens and other components. Third, it is possible to have fine control over the pitch by using a controlled polishing process, which provides enormous flexibility for modifying and optimizing the innate lens parameters such as the beam diameter (BD) at the focal point and the working distance (WD). For these reasons, GRIN lenses are very promising optical components for the construction of miniaturized OCT probes.

GRIN lens-based OCT imaging probes were constructed by attaching the GRIN lens, SMF, and other components with optical adhesive or epoxy, which requires precise central alignment and application of the optical cement on miniature components. At these size scales, the thickness of the cement could, in fact, be a significant factor affecting the optical characteristics of the probe. Furthermore, OCT probes are usually housed in a flexible and transparent hollow sheath. Thus, the fabrication procedures play very important role in accurate probe design. Proper selection of probe components and reduction of errors in the fabrication of optical probes with specific design parameters

$1083-3668 / 2010 / 15(6) / 066027 / 10 / \$ 25.00$ 
is critical. When designing imaging probes, numerous trade-offs exist between probe size, WD, and achievable lateral resolution. Although commercial optical design software is typically used for this design process and parameter selection, commercial software does not readily enable one to identify the influence of each component in the final probe performance. Therefore, a thorough theoretical analysis is required to identify the function of each component and obtain optimal probe design parameters. However, the theory of GRIN lens-based probes has either been largely overlooked or only simplified configurations of probe designs have been considered. ${ }^{7-11}$

In this paper, we numerically analyze the performance of various GRIN lens-based OCT probes constructed from commercially available components. A theoretical model is formulated by analyzing the propagation of a single-mode $\left(\mathrm{TEM}_{00}\right)$ Gaussian beam, within the paraxial approximation. Two types of probes are investigated, a short-WD probe and a long-WD probe. The performance of the short-WD probe is first characterized as components are added step by step following the fabrication procedure. The influence of the transparent outer tubing or sheath in the probe performance is also evaluated. Long-WD probe designs, including single- and dual-GRIN-lens models, are then compared and discussed.

\section{Theory}

Matrix formulation of Gaussian optics has been applied to obtain analytic expressions for the beam waist and WD for different probes. A Gaussian beam can be uniquely represented at a given transverse plane by the complex parameter: ${ }^{11,12}$

$$
q=z+i z_{0}
$$

where $z$ is the distance of the given transverse plane from the beam focus, and $z_{0}$ is the Rayleigh range. When a Gaussian beam characterized by $q_{1}$ passes through optical components represented by an $A B C D$ matrix within the paraxial approximation, the new complex parameter $\mathrm{q}_{2}$ of the beam in output plane is given by

$$
q_{2}=\frac{A q_{1}+B}{C q_{1}+D}
$$

In our formulation, we begin our analysis with a Gaussian beam at the end of a single mode fiber. It is assumed that the beam waist at this plane has a minimum value (i.e., it is at focus), so that the complex parameter can be written as

$$
q_{1}=i z_{01}=\frac{\pi n_{f} w_{0}^{2}}{\lambda}=\frac{n_{f} i}{a_{0}}=\frac{i}{a},
$$

where $z_{01}$ is the Rayleigh range of the initial Gaussian beam, and $a$ is defined as its inverse. The terms $n_{f}, w_{0}$, and $\lambda$ denote the refractive index of the fiber core, the beam radius at the fiber core, and the wavelength of the guided beam, respectively. The probe can be modeled as an $A B C D$ matrix combining sequential matrices of each component. The resulting complex beam parameter of the propagating light at the output plane is represented by

$$
q_{2}=\frac{A C+B D a^{2}}{C^{2}+D^{2} a^{2}}+i \frac{(A D-B D) a^{2}}{C^{2}+D^{2} a^{2}} .
$$

From the complex parameter just indicated, the WD $z_{w}$ can be directly calculated by taking the negative of its real part:

$$
\begin{aligned}
& z_{w}=-\frac{A C+B D a^{2}}{C^{2}+D^{2} a^{2}}, \\
& z_{02}=\frac{(A D-B D) a^{2}}{C^{2}+D^{2} a^{2}} .
\end{aligned}
$$

The imaginary part of $q_{2}$ also represents the new Rayleigh range $z_{02}$ at the focal length, which yields the beam waist at the focal length. Finally, the resulting beam waist $w_{02}$ in terms of the initial waist can be calculated as follows:

$$
w_{02}=w_{01}\left(\frac{n_{f} a z_{02}}{n_{s}}\right)^{1 / 2}=w_{01} a_{0}\left[\frac{1}{n_{s} n_{f}} \frac{(A D-B C)}{C^{2}+D^{2} a^{2}}\right]^{1 / 2},
$$

where $w_{01}$ is the initial beam waist in the input plane, and $n_{s}$ is the refractive index of the medium at the last output plane. In the following sections, we present analytic expressions for different probes.

\section{Results and Discussion}

\subsection{Single GRIN Lens-Based Probe}

The common endoscopic OCT probe has a single GRIN lens configuration combined with other components such as an optical fiber, spacer, and reflecting prism. To approach realistic investigations, dimensions and parameters of typical endoscopic probes were applied: center wavelength $(1.3 \mu \mathrm{m})$, diameter of GRIN lens ( $1 \mathrm{~mm}$ ), pitch of GRIN lens (0.26 to 0.3 ), prism size (500 $\mu \mathrm{m}, 700 \mu \mathrm{m}, 1 \mathrm{~mm}$ ), SMF (core/cladding: 9/125 $\mu \mathrm{m}$ ).

\subsubsection{Effect of spacers between fiber tip and a GRIN lens}

The initial procedure to assemble a probe is to attach an optical fiber and GRIN lens by UV adhesive or thermal fusion. The variance of the spacer in between the GRIN lens and the fiber is known to be one of the most critical factors for determining the $\mathrm{BD}$ and the WD in the probe. ${ }^{13}$ Thus, the refractive index and control of the cement thickness control in the fabrication is important to achieve an optimal design. To verify this, a single GRIN lens-probe without a prism was first evaluated, as presented in Fig. 1(a).

The BD and WD were monitored by varying the thickness of the spacer for the cases of an air gap and optical adhesive. Figure 2(a) and 2(b) show graphs of these as functions of the GRIN lens pitch. The graphs show that both the WD and BD decrease as the distance between the optical fiber and GRIN lens is increased, which is very sensitive to even a short length of spacer. This is because adding a spacer changes the incident beam parameter entering the GRIN lens. As the spacer length increases, the $\mathrm{BD}$ of the propagating light through the spacer becomes larger, which subsequently increases the BD at the input surface of the GRIN lens. This has the effect of changing 


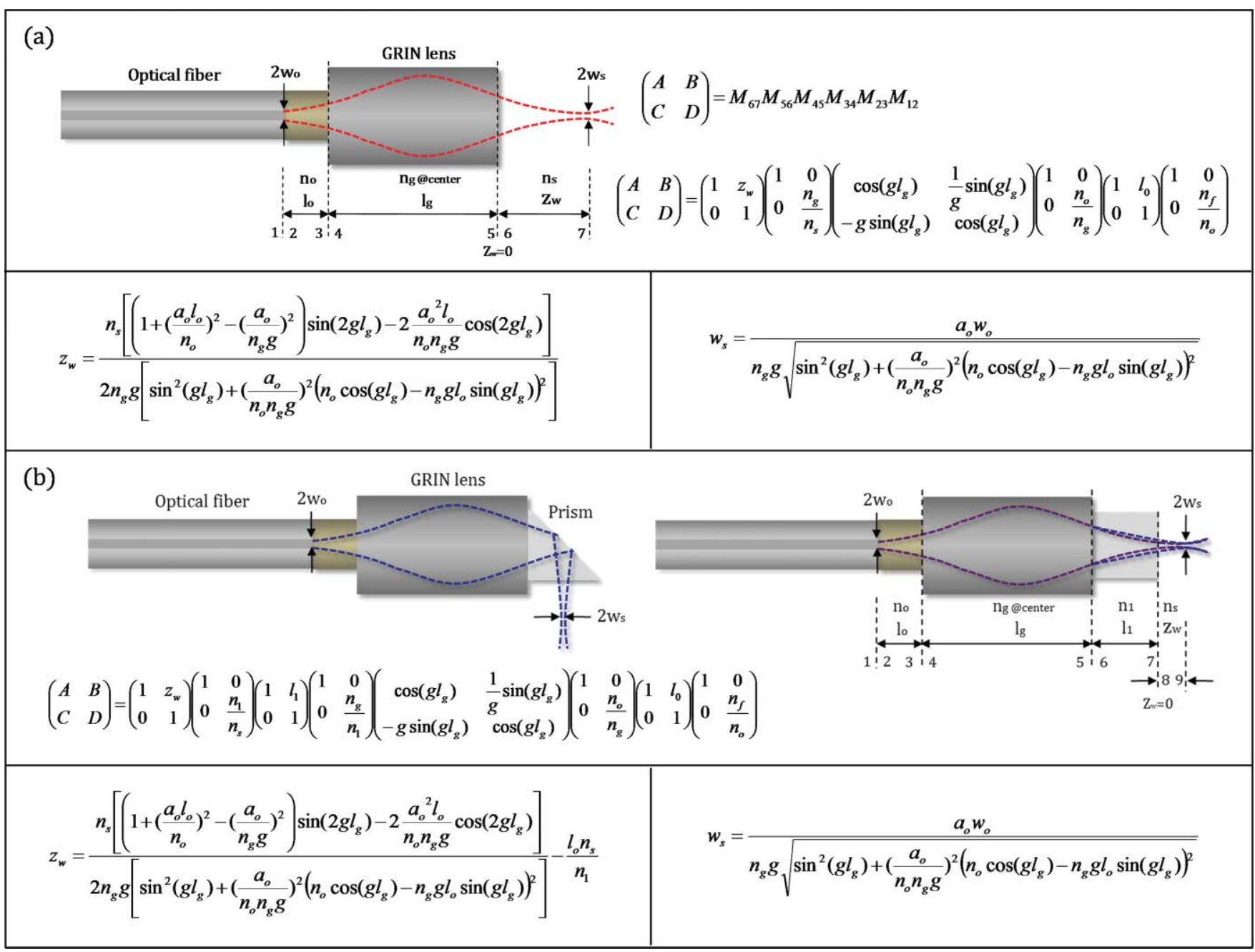

Fig. 1 Schematic diagram of a single GRIN lens-based OCT probe (a) with and (b) without a prism. Corresponding ABCD ray transfer matrix and equations of WD $\left(z_{w}\right)$ and beam radius $\left(w_{s}\right)$ are presented for each model. All calculations were performed at a wavelength of $1.3 \mu \mathrm{m}$, where $W_{o}$ : initial beam radius from the optical fiber; $n_{f}$ : the refractive index of the optical fiber; $n_{o}$ : the refractive index of spacer between an optical fiber and a GRIN lens; $I_{0}$ : length of spacer; $n_{g}$ : refractive index of GRIN lens at the center; $I_{g}$ : length of GRIN lens; $n_{1}$ : refractive index of prism $l_{1}$ : length of $45^{\circ}$ angled reflection prism; g: gradient constant of GRIN lens; $n_{s}$ : refractive index of imaged specimen.

the trajectory of light within the GRIN lens. The trajectory of propagating light within the GRIN lens has three phases: (1) divergence, (2) access to the location having the maximum BD, and (3) focus. The larger incident BD causes the beam to approach the maximum beam diameter within the GRIN lens at a shorter distance, while the distance to reach the output surface of the GRIN lens is extended. Finally, the light from the GRIN lens is focused, producing a shorter WD and a smaller BD at the focal point. For example, the light propagating out from the GRIN lens would be collimated when the ideal point source enters a 0.25 pitch GRIN lens. As the spacer is added, the collimated beam would start to focus. The WD and BD of the probe become progressively shorter and smaller as the length of the spacer is increased. This effect is less sensitive in the case of optical cement. This is because the small refractive index difference between the SMF and the cement allows for a smaller divergence angle in the spacer, and a resultant small incident BD at the input plane of the GRIN lens. This indicates that the imaging parameters of the probe change after the assembling process with optical adhesive, as would be expected. Thus, the probe design must consider the fabrication procedure including the UV cured cement thickness and refractive index to achieve the desired probe specifications.

The BD at the focal position of typical endoscopic probes is in the range of 20 to $30 \mu \mathrm{m}$. Two methods can be implemented to improve the lateral resolution in the current probe configuration: (1) increase the gradient constant of the GRIN lens and (2) use longer spacers. In both cases, the WD would decrease. GRIN lenses with higher gradient constants are commercially available, but have smaller diameters. The control of the gradient constant by keeping the diameter constant is also possible; however, this requires customized fabrication. Thus, the second method is more practical and preferred. A rigid glass rod or a coreless optical fiber is often inserted between the optical fiber and the GRIN lens to function as longer spacer, with lengths that are not possible using optical cement. Figure 2(c) shows the variation of WD and BD when a millimeter size spacer was added. With the same principle as in the previous cases of air and cement gaps, the lateral resolution was improved as the length of the spacer increased. Even though the WD was also 
Jung et al.: Numerical analysis of gradient index lens-based optical coherence tomography...
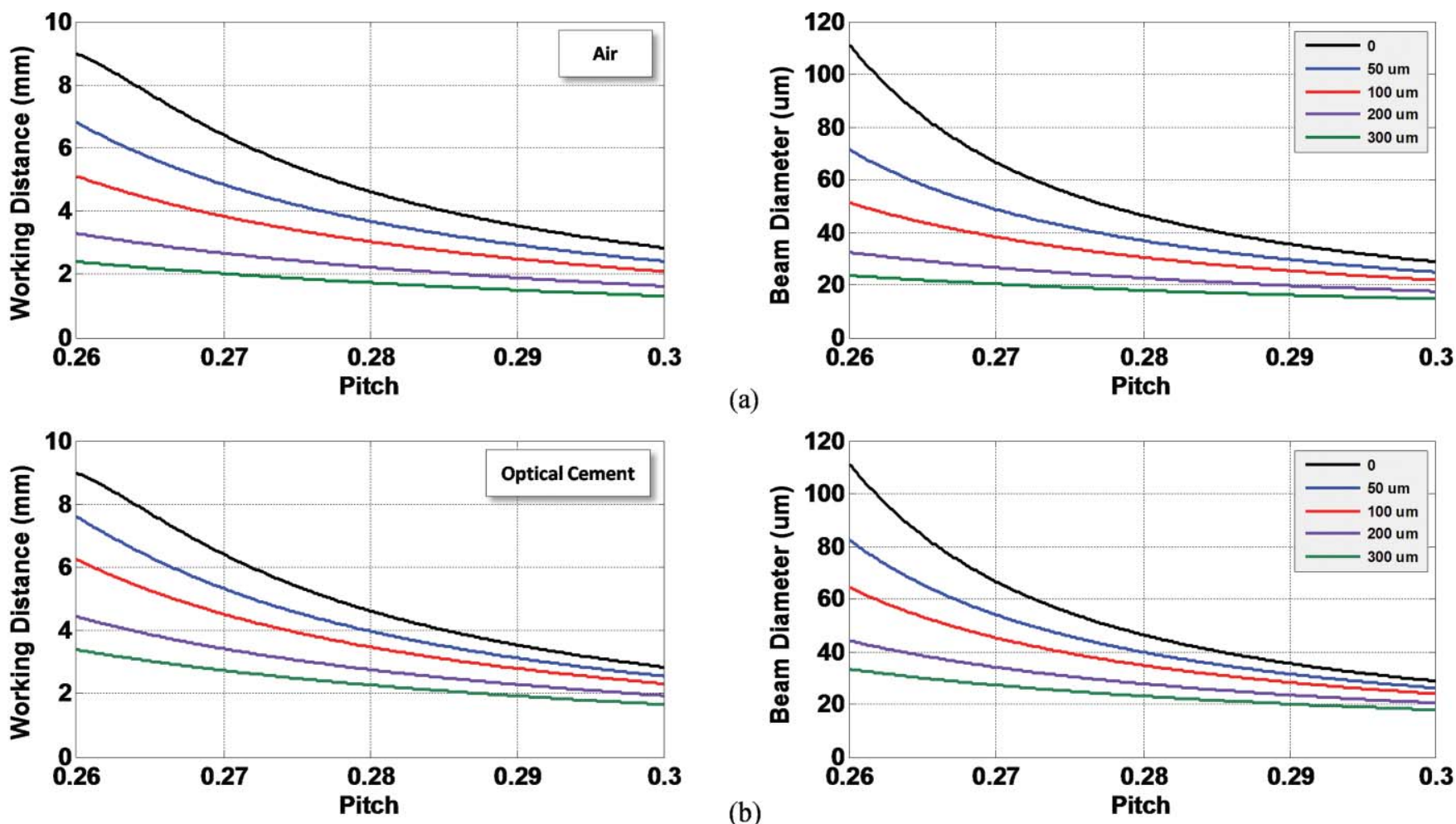

(a)
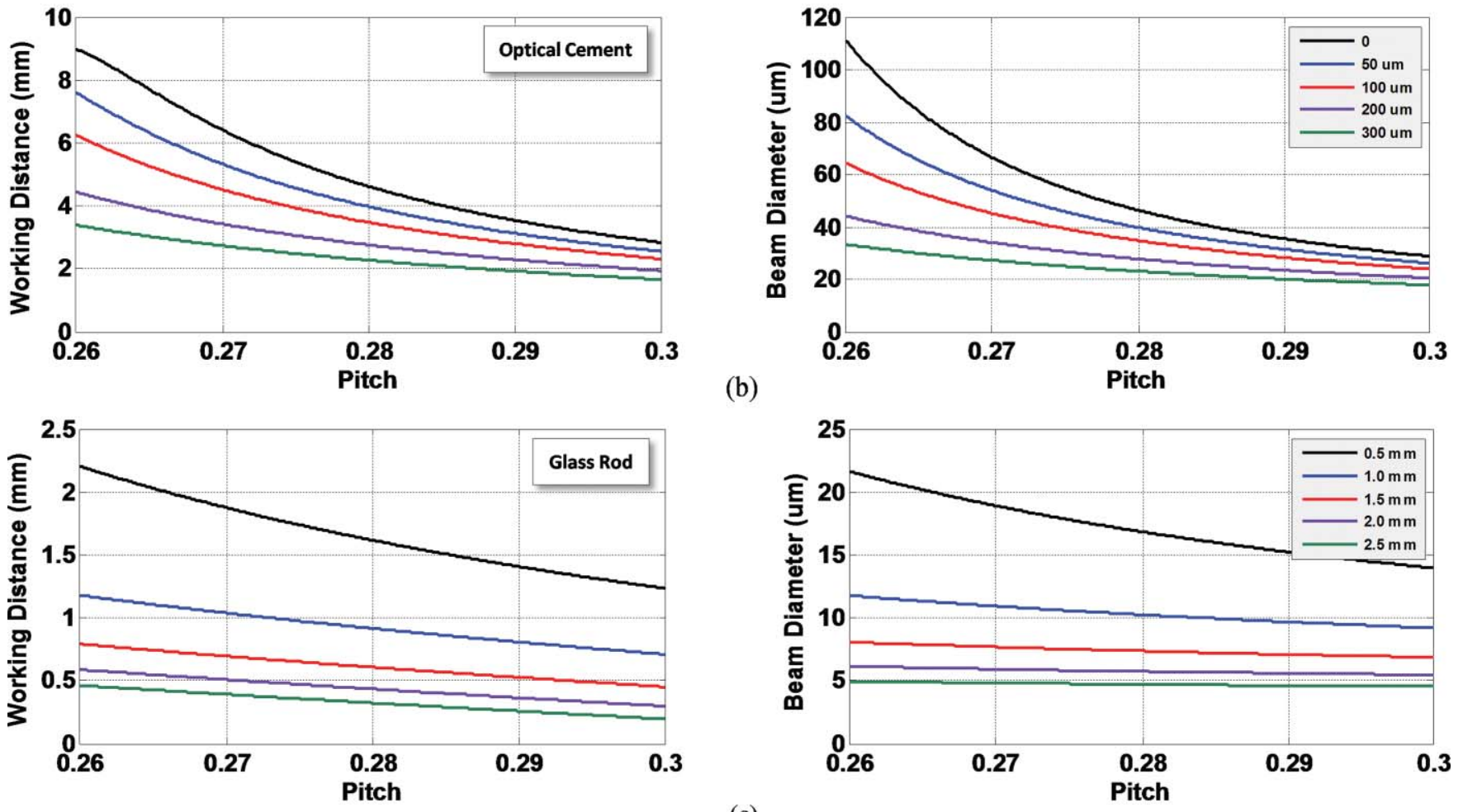

(c)

Fig. 2 WD and BD of probes for varying lengths and types of spacers including (a) air, (b) optical cement gap, and (c) glass rod. In this calculation, a $1 \mathrm{~mm}$ diameter GRIN lens (gradient constant: $0.653 \mathrm{~mm}^{-1}$ ) was considered at a $1.3 \mu \mathrm{m}$ wavelength.

significantly reduced, it is still within a useful range for endoscopic OCT imaging.

\subsubsection{Beam profile of probe by component and refractive index after a GRIN lens}

The next step in fabrication of side-imaging probes is to attach the reflecting prism to direct the beam toward the specimen, typically $90 \mathrm{deg}$ from the long axis of the fiber or endoscopic probe. Figure 3(a) shows the WD and BD after adding the prism. In this calculation, the cement thickness between the GRIN lens and prism was not considered, because the interface is always minimized in the fabrication procedure to attach the GRIN lens with the prism. In fact, adding components after the GRIN lens, such as optical cement or prism, does not have an effect on the BD, as depicted in the equations of Fig. 1. These added components only reduce the WD.
The dominant factors that determine the BD at the focal position are the focusing angle and the $\mathrm{BD}$ at the end plane of the GRIN lens. These factors are preserved as long as light propagated in a uniform material with a flat surface. However, the WD is dependent on the length of the prism as well as its refractive index, as can be seen in both Fig. 3(a) and the equations. The preceding results indicate that dynamic focusing of the probe along the imaging depth is feasible, keeping the same beam diameter at the focal position. Integration of additional components in series after GRIN lens only affects the BD. Thus, by varying the length and refractive index, one could achieve dynamic focusing. Physically, varying the length of a component is difficult, but the change of the refractive index is more feasible by means of an index-tunable material. By our calculation, a variation of $300 \mu \mathrm{m}$ in WD when using a 0.27 pitch GRIN lens would require a change in refractive index from 1.4 to 1.8 . 

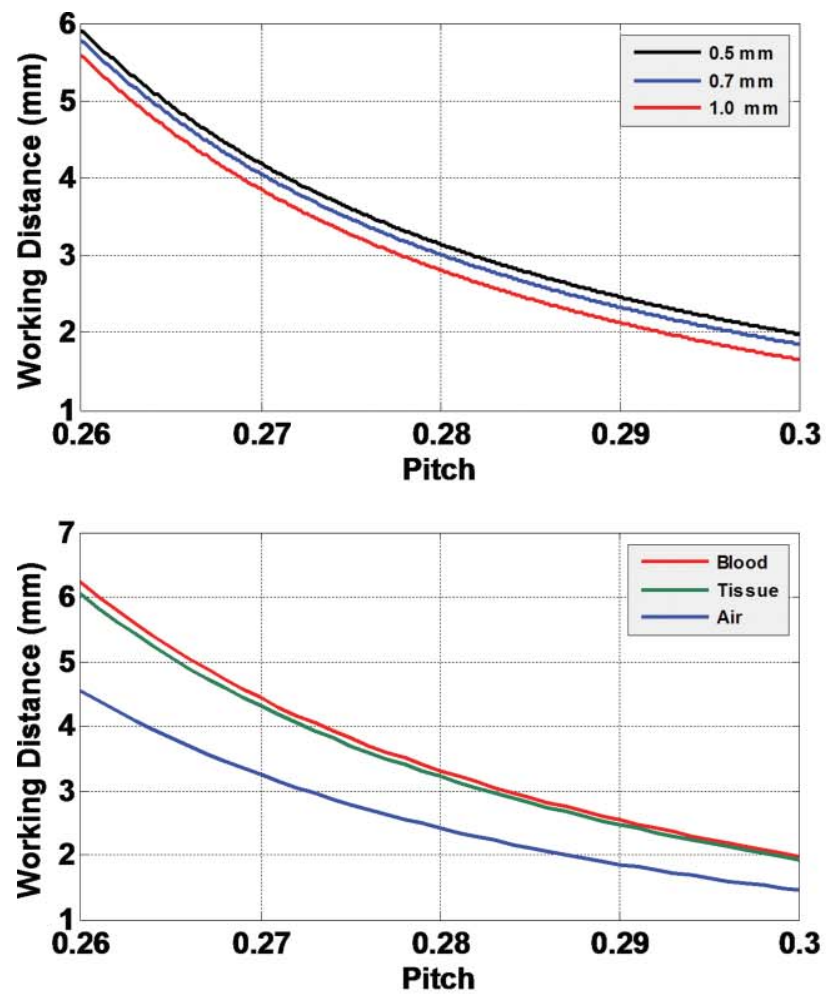

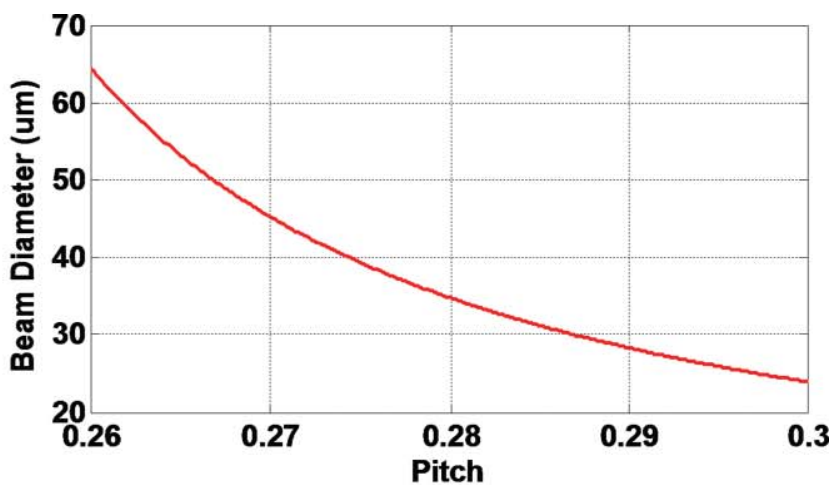

(a)

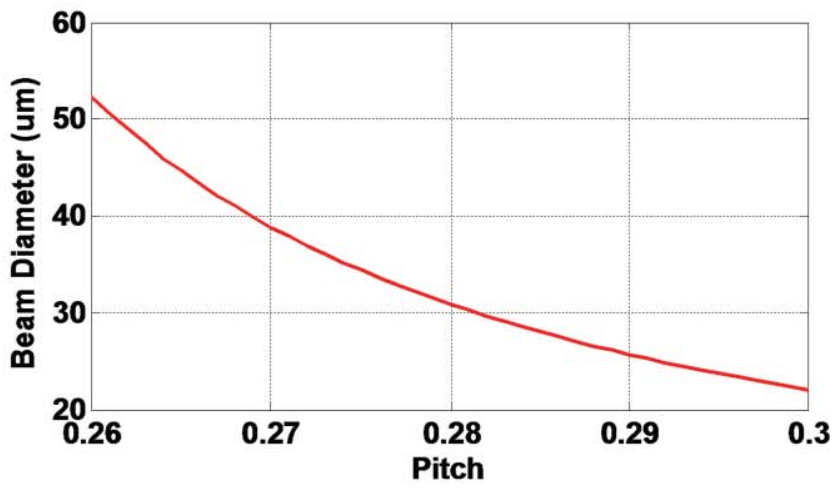

(b)

Fig. $3 \mathrm{WD}$ and BD of probes for varying (a) prism length and (b) refractive index of the sample medium. In both sets of graphs, a $100 \mu \mathrm{m}$ optical cement and a $1 \mathrm{~mm}$ diameter GRIN lens were used. Graphs in (a) were calculated in an air medium, and a 1 mm length of prism was considered for the graphs in (b).

OCT probes are often used in other media rather than air. For example, needle imaging probes are directly surrounded by tissue, while probes for cardiovascular imaging are immersed in blood or flushed with saline solutions. Figure 3(b) shows probe characteristics for different media. The graphs show that probes in higher refractive index media have longer WD, while the $\mathrm{BD}$ remains the same. If the refractive index of the propagating material has a lower refractive index compared to the prism, light will be more rapidly focused. However, for a higher refractive index medium, the light will have a smaller focusing angle. This is the reason why the WD of a probe in tissue or blood is longer than in air.

\subsubsection{Influence of tubing on the beam profile of the probe}

The last step in the fabrication process is to enclose the microoptics in a glass or plastic tubing or sheath to protect the probe and make its use more robust. Since the tubing has a cylindrical shape, its geometry works as a cylindrical lens and produces optical aberrations. ${ }^{14}$ The ideal investigation of probes with tubing would analyze the beam profile both along and across the curvature of the tubing. For simplicity, we are only considering the beam profile for the cross section view of tubing, as seen in Fig. 4.

To investigate the influence of the tubing on the probe parameters, we compared the beam profile of probe designs before and after the addition of the tubing. First, the WD and $\mathrm{BD}$ of two different probes without tubing were surveyed when used in different sample media, as shown in Table 1. The WD, $\mathrm{WD}^{*}$, and BD were then recalculated after considering the tubing parameters. WD is the distance from the outer surface of the tubing to the focal spot and $\mathrm{WD}^{*}$ is the distance from the end of the prism to the focal position; i.e., it includes $l_{2}$ (the length between prism and tubing), $l_{t}$ (the thickness of the tubing), and $Z_{w}$ (the length after tuning to the focal position). As seen in Table $1, \mathrm{WD}^{*}$ and BD have increased in both the 0.27 and 0.29 pitch probes when the tubing geometry was taken into account due to the fact that the curved structure of the tubing causes the focusing beam to diverge. This tendency is greater for a lower pitch probe, while a tightly focused beam from a higher pitch probe has less divergence. In the probe design, the confocal parameter (CP) is also an important factor because the converging and diverging Gaussian beam above and below the CP will have larger spot size diameters, and hence poorer transverse resolution. The $\mathrm{CP}$ for each different probe design was calculated by $\mathrm{CP}=2 \frac{\pi w_{S}^{2}}{\lambda_{0}}$, and compared in Table 1.

Table 1 provides useful intuition to design and fabricate GRIN lens-based OCT probes. Though the beam characteristics of probes may have the desired values without tubing, once the tubing is added, these values may change significantly. During fabrication, the central positioning of the probe within the tubing is also important to minimize any unexpected performance errors due to probe location within the tubing, which varies with bending radius and distance between the probe and tubing. Thus, these effects must be considered to obtain the optimal beam parameters of the probe. 


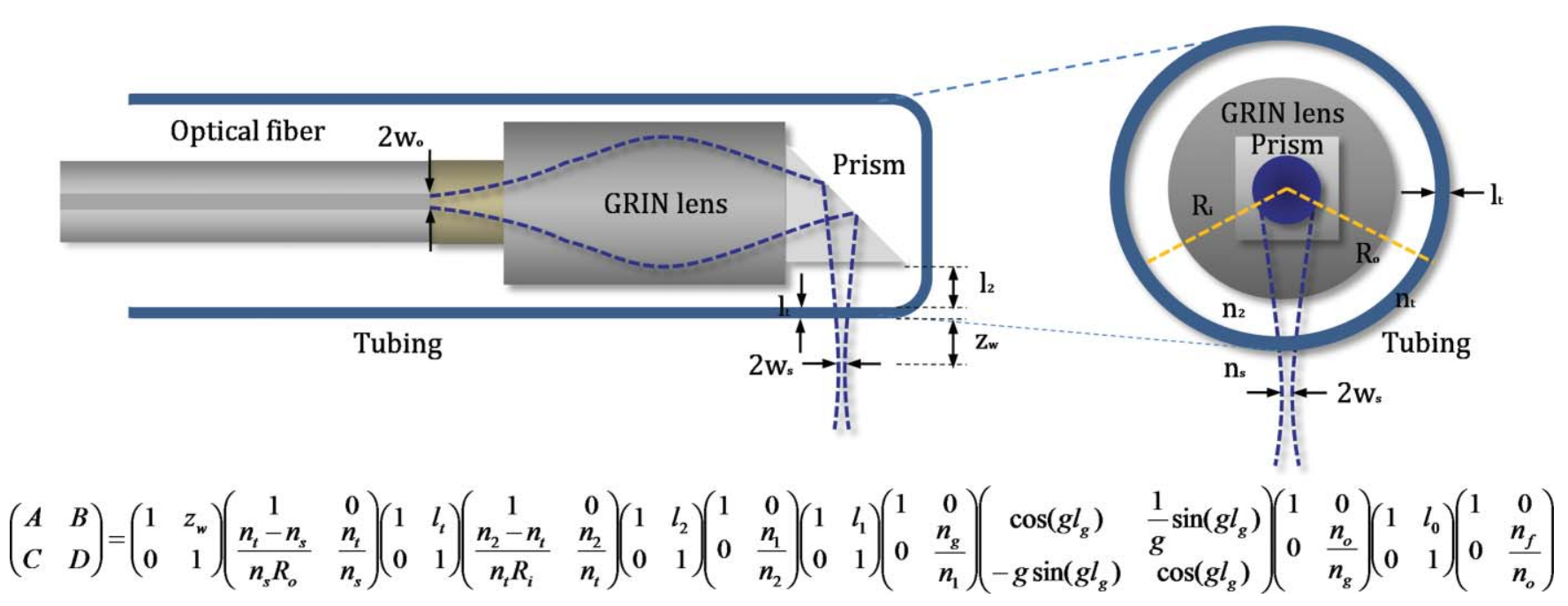

Fig. 4 Schematic and $A B C D$ ray transfer matrix of a single GRIN lens-based probe with tubing: $I_{2}$; length between prism and tubing; $n_{2} ;$ refractive index of medium between prism and tubing; $l_{t}$ : thickness of tubing; $n_{t}$ : refractive index of tubing; $R_{i}$ : inner diameter of tubing and $R_{o}$ : outer diameter of tubing.

Figure 5 shows in greater detail the effect of various tubing parameters on the beam parameters. For these calculations, the probe parameters detailed in Table 1 were used. The variation of WD* and BD with changing tubing thickness is shown in Fig. 5(c), which reveals that varying the tubing thickness does not significantly affect the beam parameters. In this configuration, the $\mathrm{WD}^{*}$ and $\mathrm{BD}$ of the probe are determined by the BD and focusing angle at the outer surface of the tubing. The focusing angle is mostly dependent on the refractive index difference between the tubing and the imaging medium, as explained previously in Fig. 3(b). The BD is determined by the tubing geometry including thickness and refractive index. In an air medium, the $\mathrm{WD}^{*}$ and BD increase because the diameter of the propagating beam through the tubing grows progressively larger as the tubing thickness is increased. However, this phenomenon has a lesser impact when imaging in other media. Even though the propagating beam in the tubing becomes larger, the influence of the tubing thickness has less effect on the probe beam profile because the small focusing angle would still be a dominating factor to determine the WD* and BD.

In all three cases in Fig. 5, a 0.29 pitch GRIN lens probe is less sensitive to tubing parameters than a 0.27 pitch GRIN lens

Table 1 Comparison of probe beam profiles before and after adding the tubing.

\begin{tabular}{|c|c|c|c|}
\hline & & 0.27 Pitch & 0.29 Pitch \\
\hline \multirow[t]{4}{*}{ Without tubing } & Probe parameters & \multicolumn{2}{|c|}{$\begin{array}{l}\text { GRIN lens (diameter: } 1 \mathrm{~mm}, \text { gradient constant: } 0.653 \mathrm{~mm}^{-1} \text {, refractive index: } 1.616 \text { ) } \\
\text { Optical cement (thickness: } 500 \mu \mathrm{m} \text { refractive index: } 1.560 \text { ) } \\
\text { Prism (length: } 700 \mu \mathrm{m} \text {, refractive index: } 1.504 \text { ) }\end{array}$} \\
\hline & in air & $\begin{array}{l}\text { WD: } 1.462 \mathrm{~mm}, \mathrm{BD}: 19.41 \mu \mathrm{m} \\
\text { CP: } 0.455 \mathrm{~mm}\end{array}$ & $\begin{array}{l}\text { WD: } 0.976 \mathrm{~mm}, \mathrm{BD}: 15.56 \mu \mathrm{m} \\
\text { CP: } 0.292 \mathrm{~mm}\end{array}$ \\
\hline & in tissue & $\begin{array}{l}\text { WD: } 1.944 \mathrm{~mm}, \mathrm{BD}: 19.41 \mu \mathrm{m} \\
\text { CP: } 0.455 \mathrm{~mm}\end{array}$ & $\begin{array}{l}\text { WD: } 1.298 \mathrm{~mm}, \mathrm{BD}: 15.56 \mu \mathrm{m} \\
\text { CP: } 0.292 \mathrm{~mm}\end{array}$ \\
\hline & in blood & $\begin{array}{l}\text { WD: } 2.003 \mathrm{~mm}, \mathrm{BD}: 19.41 \mu \mathrm{m} \\
\text { CP: } 0.455 \mathrm{~mm}\end{array}$ & $\begin{array}{l}\text { WD: } 1.337 \mathrm{~mm}, \mathrm{BD}: 15.56 \mu \mathrm{m} \\
\text { CP: } 0.292 \mathrm{~mm}\end{array}$ \\
\hline \multirow[t]{4}{*}{ With tubing } & Tubing parameters & $\begin{array}{l}\text { Inner radius of tubing: } 700 \mu \mathrm{m} \\
\text { Refractive index of tubing: } 1.35 \\
\text { Tubing thickness: } 300 \mu \mathrm{m}\end{array}$ & \\
\hline & in air & $\begin{array}{l}\text { WD: } 1.257 \mathrm{~mm}, \mathrm{BD}: 24.29 \mu \mathrm{m} \\
\text { CP: } 0.713 \mathrm{~mm} \\
W^{*}: 1.907 \mathrm{~mm}\end{array}$ & $\begin{array}{l}\text { WD: } 0.552 \mathrm{~mm}, \mathrm{BD}: 18.24 \mu \mathrm{m} \\
\text { CP: } 0.402 \mathrm{~mm} \\
W^{*}: 1.202 \mathrm{~mm}\end{array}$ \\
\hline & in tissue & $\begin{array}{l}\text { WD: } 2.591 \mathrm{~mm}, \mathrm{BD}: 40.7 \mu \mathrm{m} \\
\text { CP: } 2.000 \mathrm{~mm} \\
W^{*}: 3.241 \mathrm{~mm}\end{array}$ & $\begin{array}{l}\text { WD: } 0.865 \mathrm{~mm}, \mathrm{BD}: 22.23 \mu \mathrm{m} \\
\text { CP: } 0.597 \mathrm{~mm} \\
W^{*}: 1.515 \mathrm{~mm}\end{array}$ \\
\hline & in blood & $\begin{array}{l}\text { WD: } 2.824 \mathrm{~mm}, B D: 44.1 \mu \mathrm{m} \\
\text { CP: } 2.349 \mathrm{~mm} \\
\text { WD*: } 3.474 \mathrm{~mm}\end{array}$ & 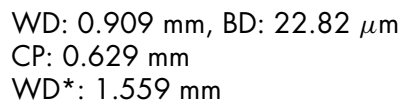 \\
\hline
\end{tabular}

$W D^{*}$ notes the distance from end surface of prism to focal length. Thus, it includes $I_{2}, I_{t}$, and $Z_{w}$ from Fig. 5 . 

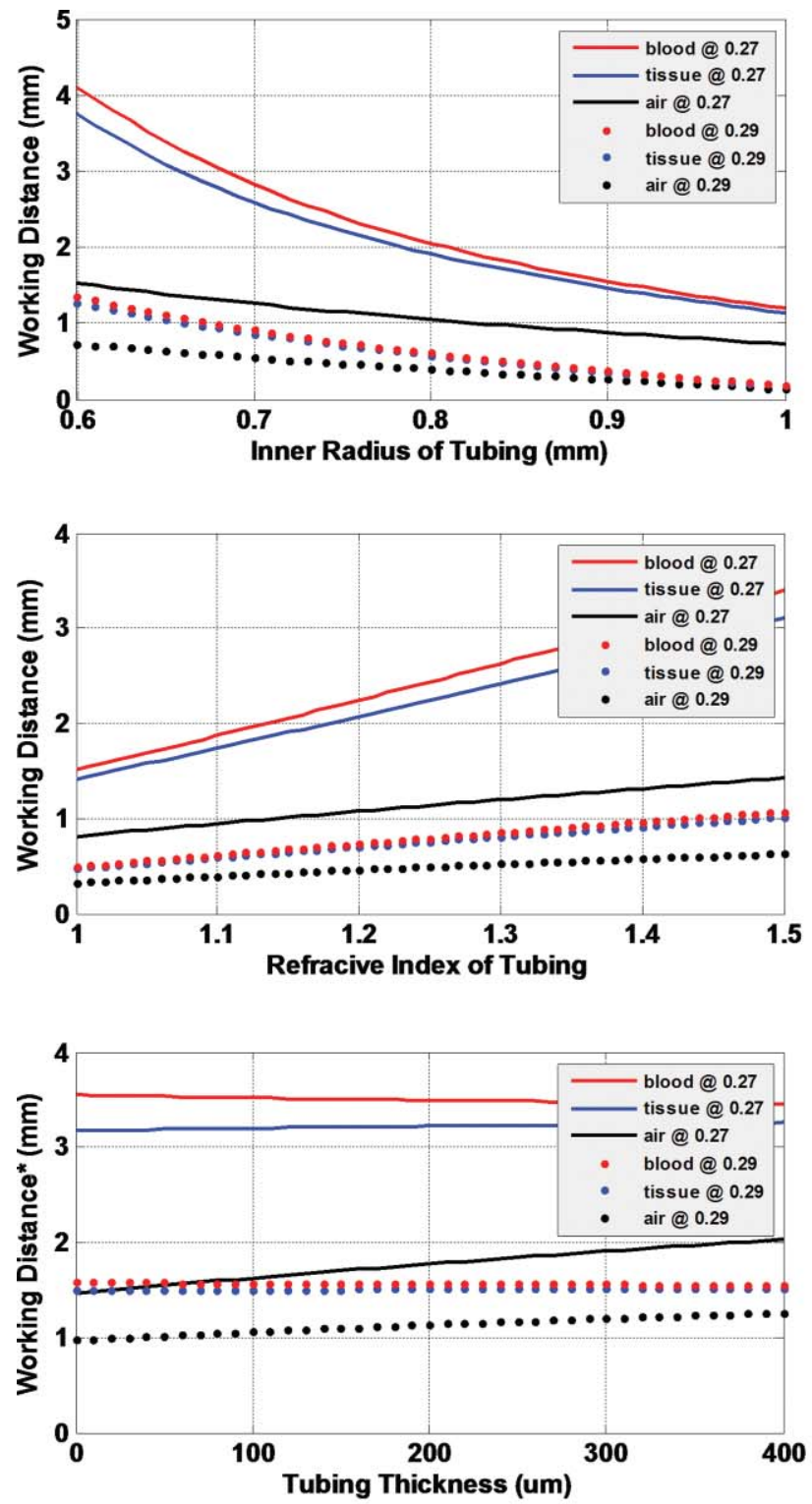

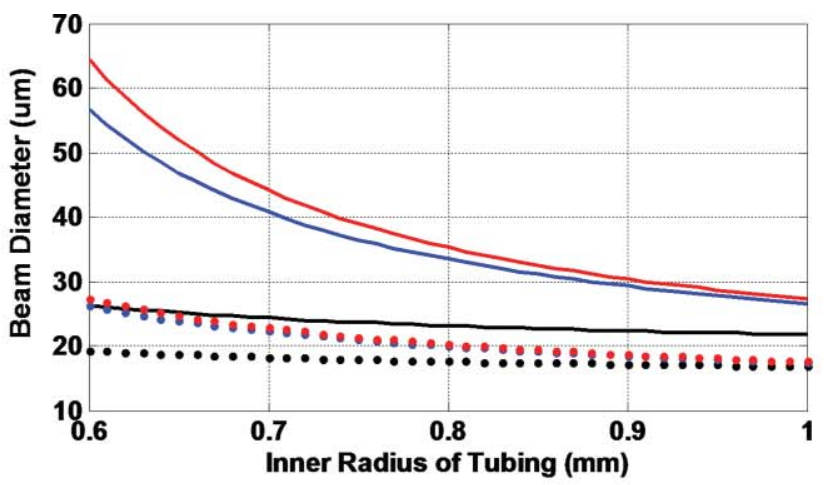

(a)

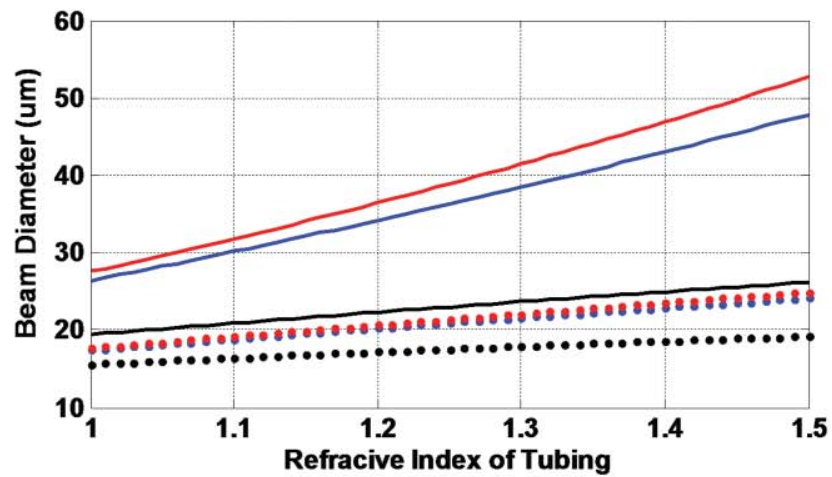

(b)

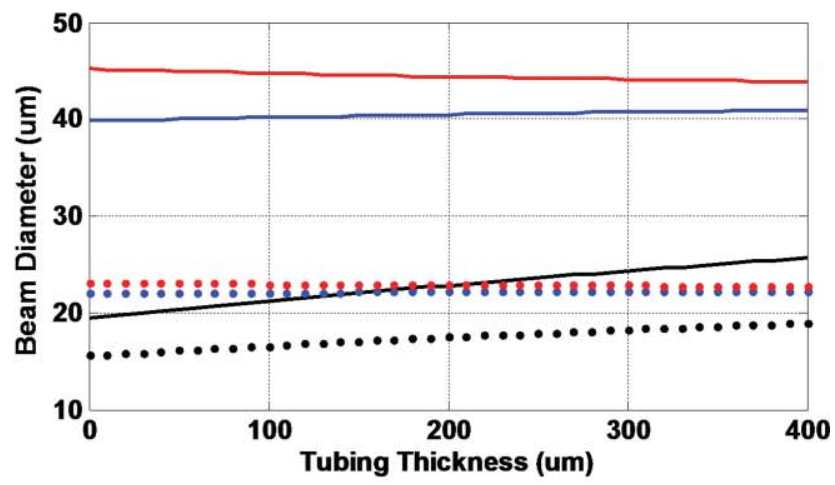

(c)

Fig. $5 \mathrm{WD}, \mathrm{WD}^{*}$ and $\mathrm{BD}$ of probes of varying tubing parameters and sample media: Plot are shown for varying (a) inner radius of tubing, (b) refractive index of tubing, and (c) tubing thickness. The design parameters and tubing specifications were as reported in Table 1.

probe. This means that a tightly focused beam with a short WD would be optimal for maintaining original beam profile and offer the best opportunity for highquality imaging after the addition of the tubing. Considering the preceding results, the material and dimensions of the selected tubing should be considered for probe design and fabrication to achieve the optimal performance for OCT imaging.

\subsection{Long Working Distance Probes}

Upto now, we have considered single GRIN lens-based OCT probes with pitches ranging from 0.26 to 0.3 . In this range, the WD and BD of the probe change proportionally to one another. Thus, with this configuration, it is difficult to design probes with a long WD and a small BD. In this section, we discuss long-WD probe designs without a prism or tubing, and with targeted beam parameters of a WD (10 to $15 \mathrm{~mm})$ and a BD (20 to $30 \mu \mathrm{m})$.
To evaluate the limitations of a typical single GRIN lens probe, the maximum BD and beam divergence angle at the end of GRIN lens was monitored, as shown in Fig. 6(a). For these calculations, the same probe design parameters were used as in Fig. 2(c). The beam profiles of these probes have a short WD (mostly less than $1.5 \mathrm{~mm}$ ) and a small BD (less than $15 \mu \mathrm{m}$ ) at the focal position. Figure 6(a) shows that the beam diameter at the output plane of a $1 \mathrm{~mm}$ GRIN lens was restricted to less than $150 \mu \mathrm{m}$. This is because the propagating light within a GRIN lens uses only a portion of the full lens diameter. In addition, the beam at the end of the GRIN lens has a tendency to focus tightly; the (-) sign in Fig. 6 indicates a focused beam. In combination, these effects result in a beam with a short WD and a small BD. Thus, it is impossible to fabricate a probe having a longer WD and a small BD in the current design.

To overcome these limitations, we chose different component parameters. First, a long glass spacer was used, which gives a 

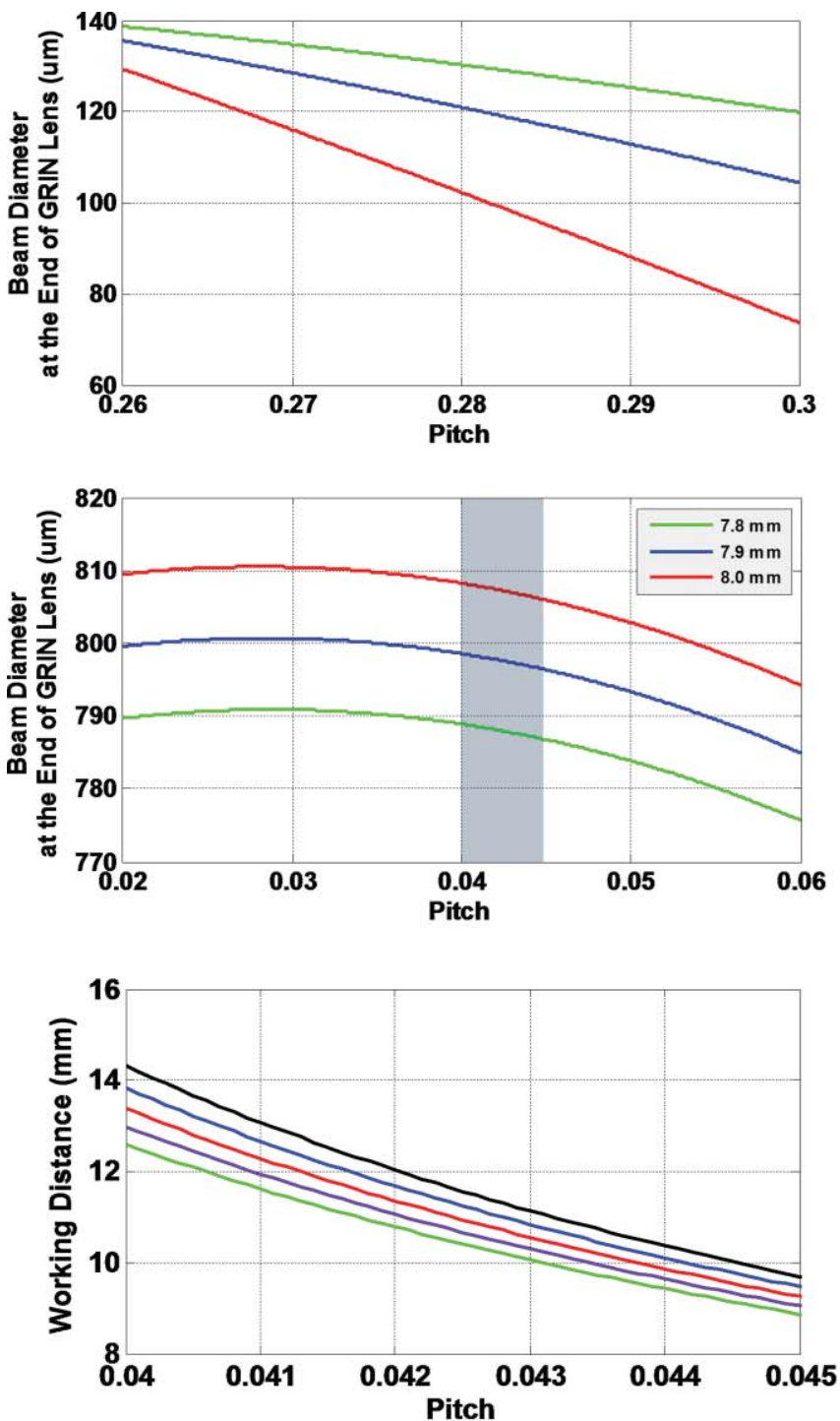

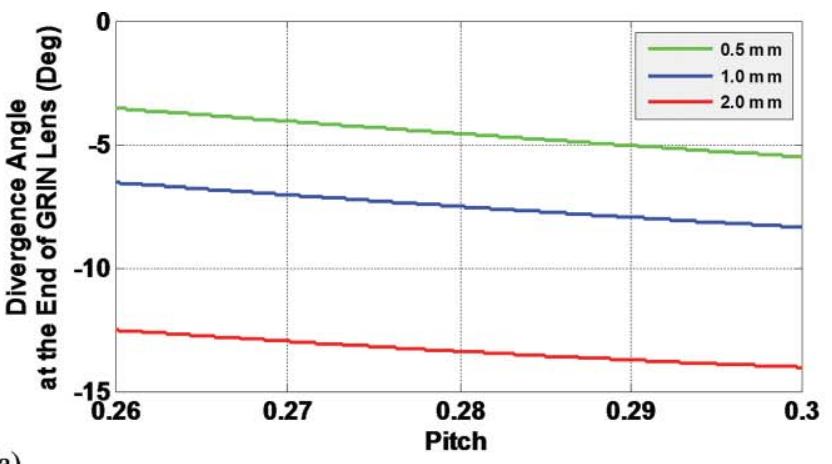

(a)

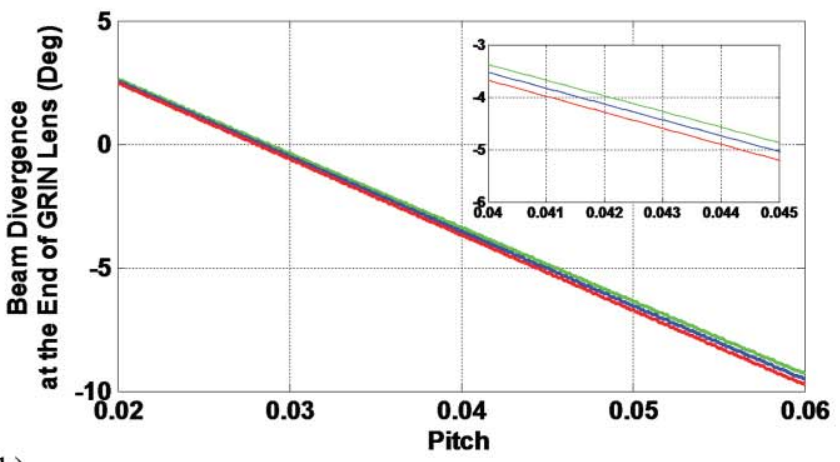

(b)

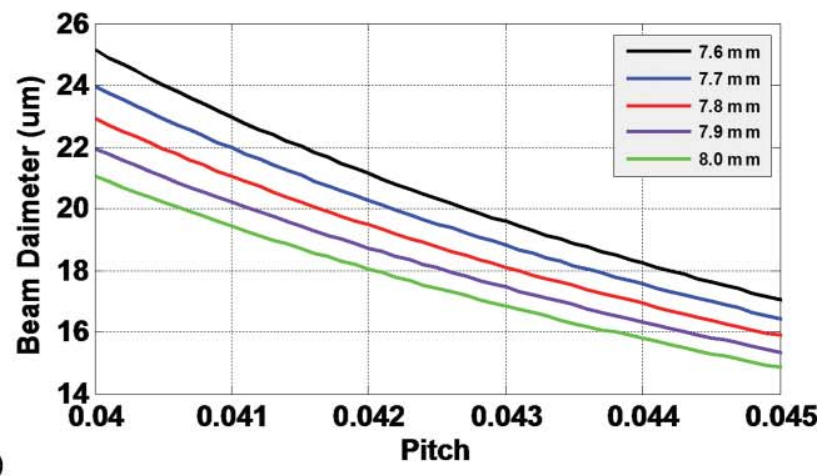

(c)

Fig. 6 Characteristics of a single GRIN lens-based probe. (a) BD and divergence angle the end of the GRIN lens for typical probes. For this calculation, the probe parameters shown in Fig. 2(c) were used. (b,c) Single GRIN lens-based probes having a longer WD and a smaller BD. The goal is to maximize the BD of the light propagating in the GRIN lens by using a longer spacer and a shorter pitch GRIN lens.

larger beam diameter at the input plane of the GRIN lens. Second, a very short pitch GRIN lens was used to change the beam focusing while maintaining a larger beam diameter. The main motivation for this probe was to maximize the numerical aperture while increasing the maximum BD of the propagating light within the GRIN lens. Based on a single GRIN lens configuration, we designed a probe with approximately an $80 \%$ maximum beam diameter, as shown in Fig. 6(b). The graphs reveal that focusing angle has increased as the beam diameter increases. With these results, we selected a pitch range from 0.04 to 0.045 and plotted the beam profile of the probe, as shown in Fig. 6(c). These graphs show these probes have the desired beam parameters, satisfying both the WD and BD requirements. In this pitch range, the $\mathrm{BD}$ was larger than the $\mathrm{BD}$ of the commonly used short-WD probe [Fig. 2(c)]. This is because the beam focusing angle ranged from -3.5 to around -5.5 , which provide less focusing, as seen in Fig. 6(a). Results show that this probe design requires a high degree of accuracy to control the length of both the spacer and the GRIN lens. Since control of the pitch of the GRIN lens requires more precision and complexity in fabrication, adjustment of the spacer length to achieve the optimal probe parameters is more desirable.

Recently, a dual GRIN lens-based probe was introduced for the same purpose. ${ }^{14,15}$ This type of probe design is shown in Fig. 7. The major objective of this design is also to maximize the BD of propagating light within the GRIN lens, which allows for a greater numerical aperture. However, the main difference between the single and dual GRIN lens-based probes is that the configuration of the dual GRIN lens probe enables one to modify the initial beam profile. A dual GRIN lens probe consists of two sets of spacers and two GRIN lenses, as depicted in Fig. 7. The first GRIN lens and spacer modify the initial beam entering the second spacer and GRIN lens. In the single GRIN lens configuration, the initial beam is determined by the beam profile of the light exiting the SMF, and cannot readily be changed. Ideally, we wish to generate a new initial beam having a smaller 


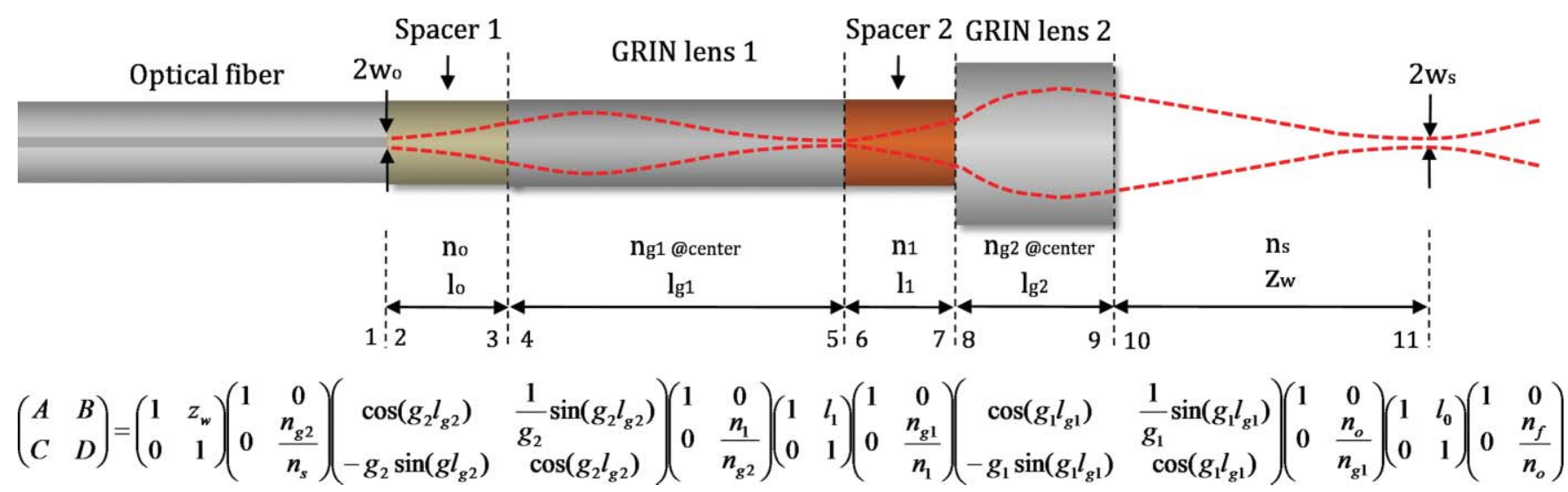

Fig. 7 Schematic diagram of a cascaded GRIN-lens-based endoscopic probe and corresponding ABCD ray transfer matrix: $n_{0}$ : refractive index of spacer 1 between an optical fiber and GRIN lens $1 ; l_{0}$ : length of spacer $1 ; n_{g} 1$ : refractive index of GRIN lens 1 at center; $l_{g}$ : length of GRIN lens 1 ; $n_{1}$ : refractive index of spacer 2 between the first and second GRIN lenses; $l_{1}$ : length of spacer $2 ; n_{g 2}$ : refractive index of GRIN lens 2 at center; $l_{g 2}$ : length of GRIN lens 2.
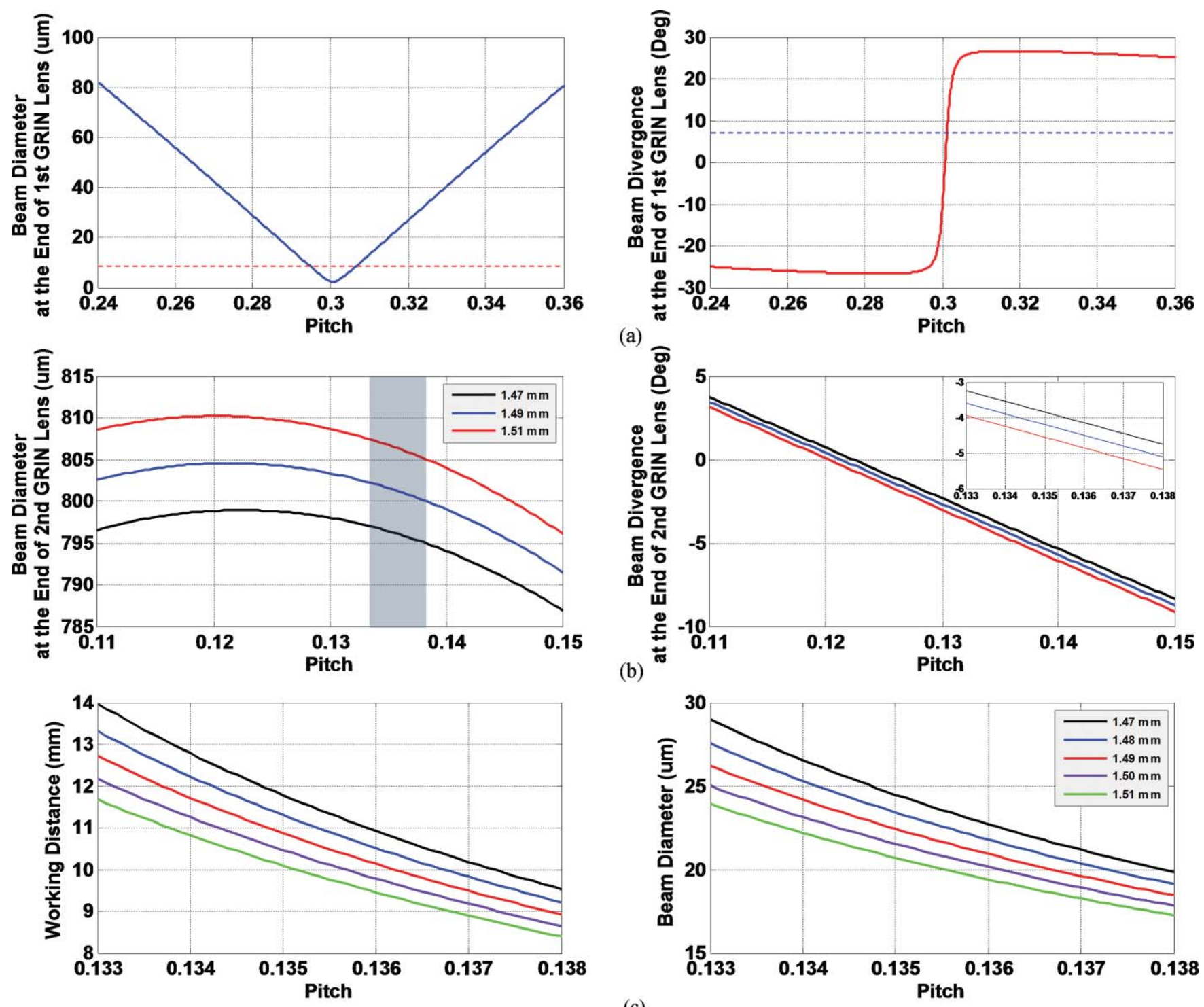

Fig. 8 Characteristics of a dual GRIN lens-based probe. (a) and (b) BD and divergence angle at the first and second GRIN lenses. (c) probe beam parameters for varying pitch ranges of the second GRIN lenses from 0.133 to 0.138 . In this calculation, the gradient constants for the first and second GRIN lens were $1.340 \mathrm{~mm}^{-1}$ and $0.653 \mathrm{~mm}^{-1}$, respectively. 
$\mathrm{BD}$ with a larger divergence angle so that light propagating through the second GRIN lens can utilize the full diameter of the GRIN lens.

We first investigated the BD and divergence angle at the end of the first GRIN lens while varying the spacer length and pitch of the first GRIN lens, to verify the characteristics and optical performance of this probe design. Figure 8(a) shows the comparison of beam parameters between a SMF (dashed line) and the combination of the first set of GRIN lens and spacer. For these calculations, we used a $500 \mu \mathrm{m}$ diam GRIN lens (gradient constant: $1.340 \mathrm{~mm}^{-1}$ ) and a $2.1 \mathrm{~mm}$ glass rod as a spacer. As seen in the left graph, a smaller BD was obtained, compared to that from a SMF, at a pitch range from 0.294 to 0.307 . This pitch range can be adjusted by varying the length of the first spacer. Under this current condition, the lowest $\mathrm{BD}$ was $2.3 \mu \mathrm{m}$ at a 0.3008 pitch.

To determine the specifications for the second set of spacer and GRIN lens, a pitch of 0.302 was selected for the first GRIN lens because this region has a higher beam divergence. The corresponding $\mathrm{BD}$ and divergence angle at the output plane of the first GRIN lens were $2.823 \mu \mathrm{m}$ and $15.73 \mathrm{deg}$, respectively. Based on these conditions, we searched for the optimal length for the second set of GRIN lens and spacer. Unlike in the single GRIN lens-based probes, the second spacer must be relatively short in length, as shown in Fig. 8(b). This is because the initial beam constructed by first set of GRIN lens and spacer has a larger divergence angle, which reduces the distance necessary to produce a larger BD of light entering the second GRIN lens. Finally, the WD, and BD at the focal position were generated for GRIN lens pitches ranging from 0.133 to 0.138 for the second GRIN lens, as shown in Fig. 8(c). In this calculation, a $1 \mathrm{~mm}$ diam GRIN lens (gradient constant: $0.653 \mathrm{~mm}^{-1}$ ) was considered for the second GRIN lens. These graphs reveal similar probe performance compared to the single GRIN lens-based probe. Thus, it is observed that both probe configurations improve the $\mathrm{BD}$ at a long $\mathrm{WD}$, which cannot be achieved with the typical single GRIN lens-based probe having pitches range from 0.26 to 0.3 .

As mentioned, the first spacer and GRIN lens in the dual GRIN lens probe generate different initial beams compared to one from SMF, but this will make the fabrication more complex. In addition, a single GRIN lens probe requires length control of the order of $100 \mathrm{~mm}$ for both the spacer and the GRIN lens, as observed in Fig. 6(b), while a dual GRIN lens probe requires a precision of the order of $10 \mu \mathrm{m}$ in spacer length, as observed in Fig. 8(b). Overall, long WD probes using a dualGRIN-lens configuration theoretically provide more freedom to produce various beam profiles due to the flexibility of modifying the initial beam. However, single GRIN lens configuration for long WD probes can construct similar performance with easier fabrication.

\section{Conclusion}

We numerically investigated GRIN lens-based OCT probe designs. The WD of typical probes changes proportionally to the BD. It was shown that the length of a spacer and the pitch of a GRIN lens both affect the WD and BD, but the components after the prism and sample medium influence only the WD. The probe properties also change significantly after taking into considera- tion the geometry of the external tubing or sheath. In all probe models, higher refractive index media such as saline, tissue, and blood make the WD longer and the BD larger. Single and dual GRIN lens probes were considered for the purpose of designing a long-WD probe. To increase WD while keeping the BD small, the BD within the GRIN lens was maximized. In both configurations, similar probe performance could be achieved. Theoretically, the dual GRIN lens probe has an advantage over the single GRIN lens probe because it can provide greater freedom in tailoring the initial beam parameters. However, it is more complex and requires greater precision during fabrication. We believe these numerical results can help facilitate the design and optimization of GRIN-lens-based OCT imaging probes for specific applications while minimizing fabrication errors.

\section{Acknowledgment}

This research was supported in part by grants from the National Institutes of Health (NIBIB, R01 EB005221 and NCI, R21/R33 CA115536, S.A.B.). Additional information can be found at http://biophotonics.illinois.edu.

\section{References}

1. D. Huang, E. A. Swanson, C. P. Lin, J. S. Schuman, W. G. Stinson, W. Chang, M. R. Hee, T. Flotte, K. Gregory, C. A. Puliafito, and J. G. Fujimoto, "Optical coherence tomography," Science 254, 1178-1181 (1991).

2. A. M. Zysk, F. T. Nguyen, A. L. Oldenburg, D. L. Marks, and S. A Boppart, "Optical coherence tomography: a review of clinical development from bench to bedside," J. Biomed. Opt. 12, 1403-1-051403-21 (2007).

3. G. J. Tearney, M. E. Brezinski, B. E. Bouma, S. A. Boppart, C. Pitris, J. F. Southern, and J. G. Fujimoto, "In vivo endoscopic optical biopsy with optical coherence tomography," Science 276, 2037-2039 (1997).

4. D. C. Adler, Y. Chen, R. Huber, J. Schmitt, J. Connolly, and J. G. Fujimoto, "Three-dimensional endomicroscopy using optical coherence tomography," Nature Photo. 1, 709-716 (2007).

5. J. M. Zara and C. A. Lingley-Papadopoulos, "Endoscopic OCT approaches toward cancer diagnosis," IEEE. J. Select. Top. Quantum Electron. 14, 70-81 (2008).

6. Z. Yaqoob, J. Wu, E. J. McDowell, X. Heng, and C. Yang, "Method and applications areas of endoscopic optical coherence tomography," J. Biomed. Opt. 11, 063001-1-063001-19 (2006).

7. W. L. Emkey, and C. A. Jack, "Analysis and evaluation of graded-index fiber lenses," IEEE. Light Wave Technol. 5, 1156-1164 (1987).

8. R. R. Khan, H. S. Dhadwal, and K. Suh, "Design and characterization of coherent integrated fiber-optic imaging probes," Appl. Opt. 33, 58755881 (1994).

9. M. V. Buren, and N. A. Riza, "Foundations for low-loss fiber gradientindex lens pair coupling with the self-imaging mechanism," Appl. Opt. 42, 550-565 (2003).

10. Y. Mao, S. Chang, S. Sherif, and C. Flueraru, "Graded-index fiber lens proposed for ultrasmall probes used in biomedical imaging," Appl. Opt. 46, 5887-5894 (2007).

11. W. T. Silfvast, Laser Fundamentals, Cambridge University Press, Cambridge UK (2003).

12. C. Gomez-Reino, M. V. Perez, and C. Bao, Gradient-Index Optics Springer, New York (2002).

13. G. J. Tearney, "Optical biopsy of in vivo tissue using optical coherence tomography," PhD Thesis, Massachusetts Institute of Technology (1997).

14. J. Xi, L. Huo, Y. Wu, M. J. Cobb and J. H. Hwang, and X. Li, "Highresolution OCT balloon imaging catheter with astigmatism correction," Opt. Lett. 34, 1943-1945 (2009).

15. H. L. Fu, Y. Leng, M. J. Cobb, K. Hsu, J. H. Hwang, and X. Li, "Flexible miniature compound lens design for high-resolution optical coherence tomography balloon imaging catheter," J. Biomed. Opt. 13, 060502 (2008). 